\title{
ULTRA-HIGH SPATIAL RESOLUTION UAV-BASED IMAGERY TO PREDICT BIOMASS IN TEMPERATE GRASSLANDS
}

\author{
U. Lussem ${ }^{1 *}$, A. Bolten ${ }^{1}$, J. Menne ${ }^{1}$, M. L. Gnyp ${ }^{2}$, G. Bareth ${ }^{1}$ \\ ${ }^{1}$ Institute of Geography, GIS \& Remote Sensing Group, University of Cologne, Albertus-Magnus-Platz, 50923 Cologne, Germany - \\ (ulrike.lussem, a.bolten, g.bareth)@uni-koeln.de, jmenne@gmx.de \\ ${ }^{2}$ Research Center for Crop Nutrition Hanninghof, Yara International ASA, 48249 Dülmen, Germany - martin.gnyp@yara.com
}

KEY WORDS: RGB, Multispectral, Vegetation Index, UAV, Biomass, Grassland

\begin{abstract}
:
Monitoring biomass yield in grassland is of key importance to support sustainable management decisions. Especially the high spatiotemporal variety in grasslands requires rapid and cost-efficient data acquisition with a high spatial and temporal resolution. Therefore, this study aims to evaluate the comparability of UAV-based simultaneously acquired vegetation indices from a consumer-grade RGBcamera (Sony Alpha 6000) and a well-calibrated narrow-band multispectral camera (MicaSense RedEdge-M) to estimate dry matter biomass yield. The study site is an experimental grassland field in Germany with four nitrogen fertilizer levels. Biomass yield and UAV-based data for the first cut in May 2018 was analysed in this study. From the RGB-data the Plant Pigment Ratio Index (PPR) and the Normalized Green Red Difference Index (NGRDI) and from the multispectral data the Normalized Difference Vegetation Index (NDVI) are calculated as predictors for dry biomass yield. The NGRDI and NDVI perform moderately well with crossvalidation $R^{2}$ of 0.57 and 0.63 respectively, while the PPR performs better with an $R^{2}$ of 0.70 . These results indicate the potential of low-cost UAV-based methods for rapid assessment of grasslands.
\end{abstract}

\section{INTRODUCTION}

Grasslands cover $40 \%$ of the earth's terrestrial surface and are of ecological and economic importance (FAO, 2010). Besides providing ecosystem functions such as carbon sequestration, grasslands are the basis of milk and meat production, but also play a role in biofuel production (O'Mara, 2012) Monitoring biomass yield throughout the growing season is of key importance to support management decisions on grasslands. Especially on intensely managed grasslands, where nitrogen fertilizer and manure are applied regularly, precision agriculture applications are beneficial to support sustainable, site-specific management decisions on fertilizer treatment, grazing management and yield forecasting to mitigate potential negative impacts. To support these management decisions, timely and accurate information is needed on plant parameters with a high spatial and temporal resolution. However, in highly heterogeneous plant communities such as grasslands, assessing their in-field variability non-destructively to determine e.g. adequate fertilizer application still remains challenging (Schellberg and Verbruggen, 2014). Especially biomass or yield estimation, as an important parameter in assessing grassland quality and quantity, is rather laborious. Biomass yield is mostly measured manually by clipping, height sticks, rising plate meters or ultrasonic sensors, handheld or vehicle-mounted (Wachendorf, 2017). Thus, the in-field variability cannot be assessed for the entire field or only with potential disturbances and the measurements are prone to subjective judgements.

The rapid development in platform and sensor technology, e.g. miniaturization and cost-efficiency of multispectral cameras and more user-friendly platforms (UAVs), open up a new spatial scale for environmental and agricultural studies and offer a cost efficient and near-real time assessment of biomass yield with high temporal and spatial resolution (Aasen et al., 2018).
Vegetation indices derived from aerial RGB images to predict biomass in crops have been investigated in only a few studies (e.g. Bendig et al. 2015, Hunt et al. 2005, Jannoura et al. 2015). Recent studies deployed UAV-based multispectral or RGB cameras to assess grassland biomass with good results (Lussem et al., 2018; Viljanen et al., 2018).

In this contribution we want to evaluate simultaneously acquired RGB-based vegetation indices from a consumer-grade RGBcamera and a well-calibrated narrow-band multispectral camera to estimate dry biomass yield on an experimental grassland field in Germany.

\section{MATERIAL \& METHODS}

The study site was established in 2017 on a conventionally managed grassland field $\left(0.5 \mathrm{ha}^{-1}\right)$ in Germany (Bergisches Land region, North Rhine-Westphalia). The fertilizer treatments $(0,50$, $100,150 \mathrm{~kg} \mathrm{~N} \mathrm{ha}^{-1}$, subsequently N1, N2, N3, N4) are applied in a chessboard-like pattern with 39 replicates per treatment, resulting in 156 plots of $36 \mathrm{~m}^{2}$. The cutting regime of three cuts per year was kept according to the local farming pattern. For this contribution we will focus on the second sampling date (May 25) of the first growth in 2018. Aerial images were acquired simultaneously with a Micasense RedEdge-M multispectral camera (MicaSense Inc., Seattle, WA, USA) and a Sony Alpha 6000 camera The MicaSense RedEdge-M camera is equipped with five distinct cameras (each 1.2 Megapixel, $47.2^{\circ} \mathrm{HFOV}$ ) in the visible to near-infrared region (Table 1). Additionally, the camera has a downwelling light sensor (irradiance sensor) and a GPS. Prior and directly after the flight a grey reference panel was captured with the MicaSense RedEdge-M.

\footnotetext{
* Corresponding author
} 


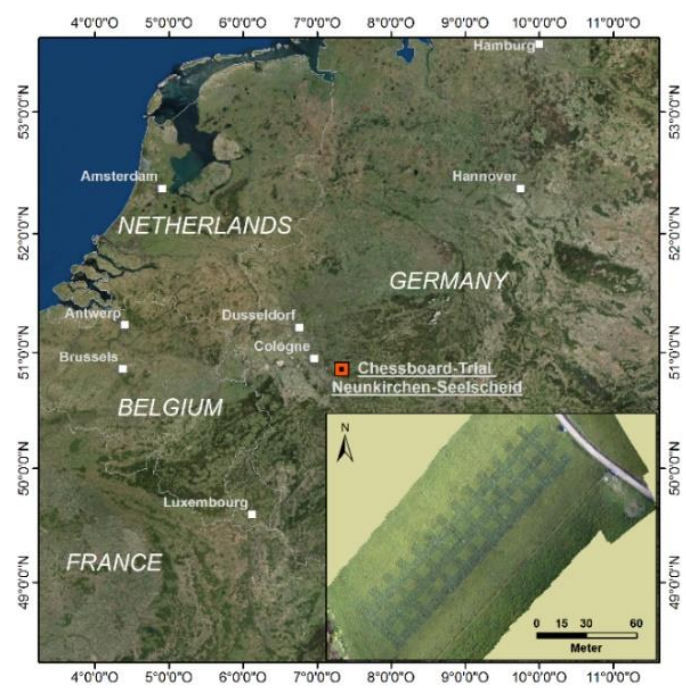

Figure 1. Location of test-site (data source: ESRI Basemaps, Eurostat). Inset map: Orthomosaic of test site.

\begin{tabular}{lcc}
\hline Band name & $\begin{array}{c}\text { Center Wavelegth } \\
{[\mathbf{n m}]}\end{array}$ & $\begin{array}{c}\text { Bandwidth } \\
{[\mathbf{n m}]}\end{array}$ \\
\hline Blue & 475 & 20 \\
Green & 560 & 20 \\
Red & 668 & 10 \\
Red Edge & 717 & 10 \\
Near Infrared & 840 & 40 \\
\hline
\end{tabular}

Table 1. Band specifications of the MicaSense RedEdge-M camera.

The Sony Alpha 6000 is a standard RGB-camera (24 Megapixel). Both cameras were mounted simultaneously on a multirotor UAV (MK Oktokopter XL with 2-axis gimbal). The flying height was set to $35 \mathrm{~m}$ above ground level resulting in a ground sampling distance (GSD) of $\sim 2.3 \mathrm{~cm}$ for the RedEdge-M, and $0.7 \mathrm{~cm}$ for the Sony Alpha 6000 . Images were acquired around 11 a.m. with stable illumination conditions.

For precise georeferencing of the images 15 ground control points (GCPs) were distributed across the test-site and measured with an RTK-GPS (Topcon GR5).

Image data was processed in the Structure-from-Motion software Photoscan v1.4 (Agisoft LLC, St. Petersburg, Russia). At least 10 markers per GCP were placed. After image alignment (high quality setting) the dense cloud was computed (high quality setting with mild depth filtering). The Micasense RedEdge-M dataset was calibrated by the calibrate reflectance function in Photoscan, using the calibration factors of the irradiance sensor and the grey reference panel. Subsequently one orthomosaic per sensor was exported with a spatial resolution of $2.5 \mathrm{~cm}$.

Index calculation was performed in ArcGIS Pro v2.2 (ESRI, Redlands, CA, USA).

\begin{tabular}{l|c|l} 
VI Name & Equation & Reference \\
\hline PPR & $\begin{array}{c}(\mathrm{G}-\mathrm{B}) / \\
(\mathrm{G}+\mathrm{B})\end{array}$ & Metternicht 2003 \\
\hline NGRDI & $\begin{array}{c}(\mathrm{G}-\mathrm{R}) / \\
(\mathrm{G}+\mathrm{R})\end{array}$ & Tucker 1979 \\
\hline NDVI & $\begin{array}{c}(\mathrm{NIR}-\mathrm{R}) / \\
(\mathrm{NIR}+\mathrm{R})\end{array}$ & Rouse et al. 1974 \\
\hline
\end{tabular}

Table 2. Vegetation indices used in this study. $\mathrm{B}=$ blue band, $\mathrm{G}$ $=$ green band, $\mathrm{R}=$ red band, NIR = near infrared band .
From the RGB-based orthomosaic the Plant Pigment Ratio (PPR) and Normalized Green Red Difference Index (NGRDI) were derived and from the multispectral orthomosaic the well-known Normalized Difference Vegetation Index (NDVI).

The indices were averaged per plot by calculating zonal statistics using a polygonal shapefile for each plot with an inside buffer of $0.5 \mathrm{~m}$ to account for border effects. Biomass samples (n: 156) were upscaled to dry matter yield $\mathrm{kg} \mathrm{ha}^{-1}$. Linear Regression analysis was performed to test the relationship between dry matter yield and the above-mentioned vegetation indices. Analysis was performed in the statistical computation software $\mathrm{R}$ v3.5 (R Core Team 2013). To assess the predictive performance of the VIs, leave-one-out cross-validation was implemented in the regression analysis using the caret-package (Kuhn et al., 2018). Prediction accuracy was quantified by calculating the coefficient of determination $\left(\mathrm{R}^{2}\right)$ and root mean squared error (RMSE).

\section{RESULTS}

Table 3 displays the descriptive statistics for dry biomass yield in $\mathrm{kg} / \mathrm{ha}$. The response of biomass to the fertilizer treatments is clearly distinguishable, although the variety between N3 and N4 is less pronounced.

\begin{tabular}{cccccc}
\hline \multicolumn{5}{c}{ Dry biomass yield [kg/ha] } \\
\hline & Mean & Median & Min & Max & SD \\
\hline N1 & 1199.56 & 1164.59 & 706.82 & 2051.55 & 247.98 \\
N2 & 1990.20 & 1987.08 & 1340.77 & 2462.87 & 217.10 \\
N3 & 2578.52 & 2523.47 & 2077.27 & 3375.84 & 278.80 \\
N4 & 2789.59 & 2813.01 & 2108.69 & 3425.19 & 312.39 \\
\hline
\end{tabular}

Table 3. Descriptive statistics of dry biomass yield for each treatment (SD: standard deviation).

A more detailed view of the orthomosaics is provided in Figures 2 and 3 . The pattern of the treatments is clearly visible, also the high similarity of treatments $\mathrm{N} 3$ and N4.

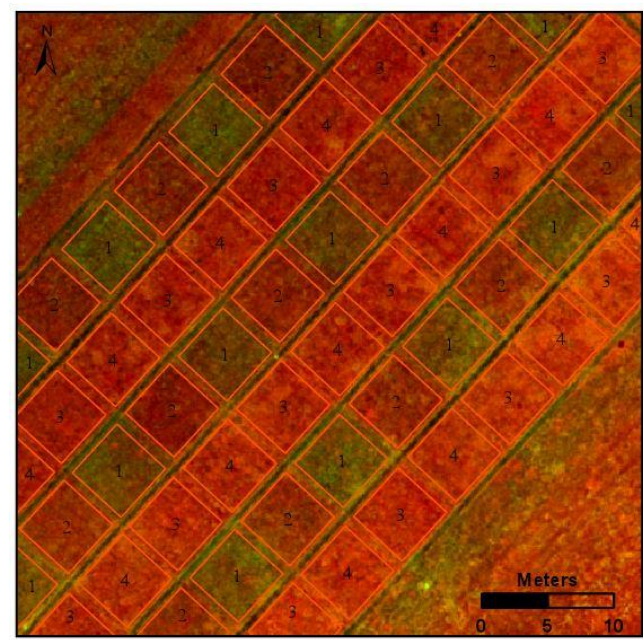

Figure 2. Detailed view of Micasense RedEdge-M based orthomosaic (band order 5-4-3). Numbers inside polygons indicate treatments. 


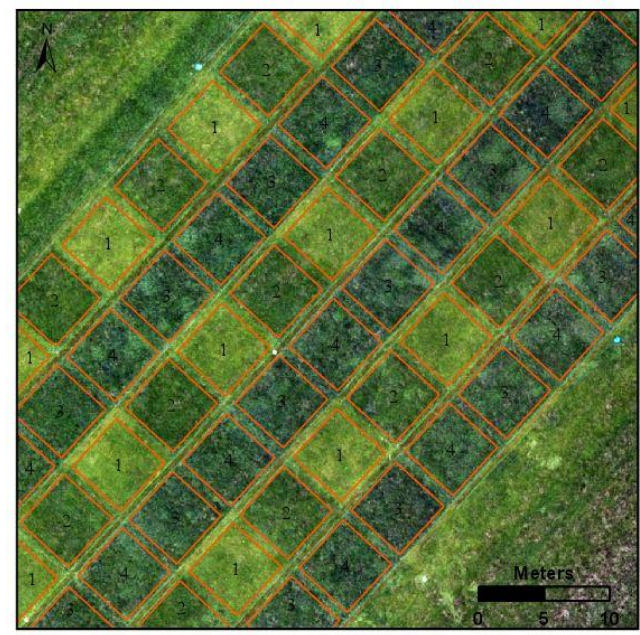

Figure 3. Detailed view of Sony Alpha 6000 based orthomosaic. Numbers inside polygons indicate treatments.

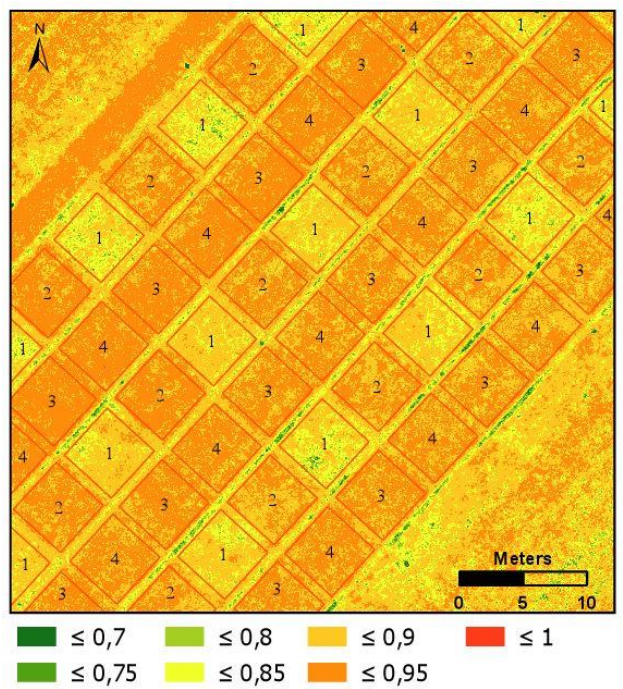

Figure 4. Detailed view of Micasense RedEdge-M based NDVI map.

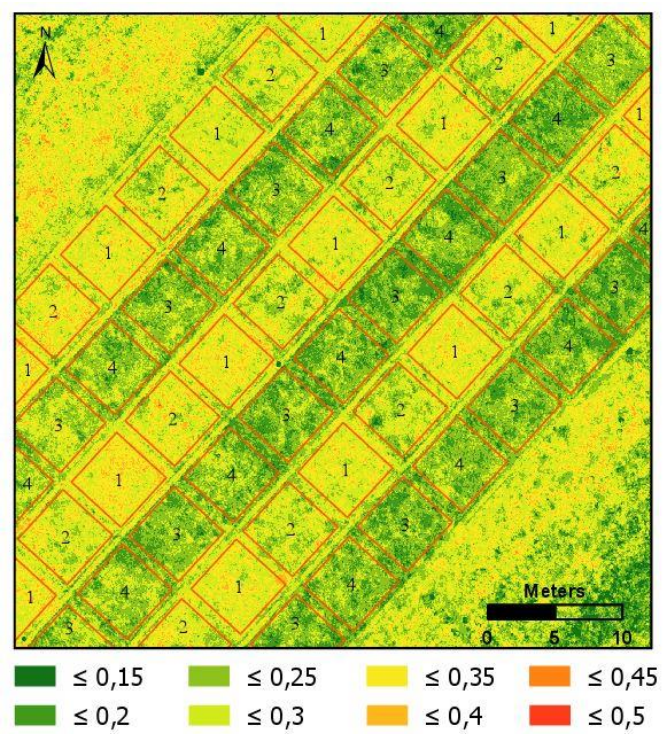

Figure 5. Detailed view of Sony Alpha based PPR map.
In Figure 4 and Figure 5 a more detailed view of the NDVI and NGRDI maps are displayed. The treatment pattern is less pronounced in the NDVI map compared to the NGRDI map. For the RGB-based PPR the values are reversed in comparison to the visual appearance and DBY. Higher biomass values correspond to lower index values. The spatial pattern of the treatments is more pronounced in the PPR map.

The relationship of NDVI and PPR to dry biomass yield is depicted in Figures 6 and 7.

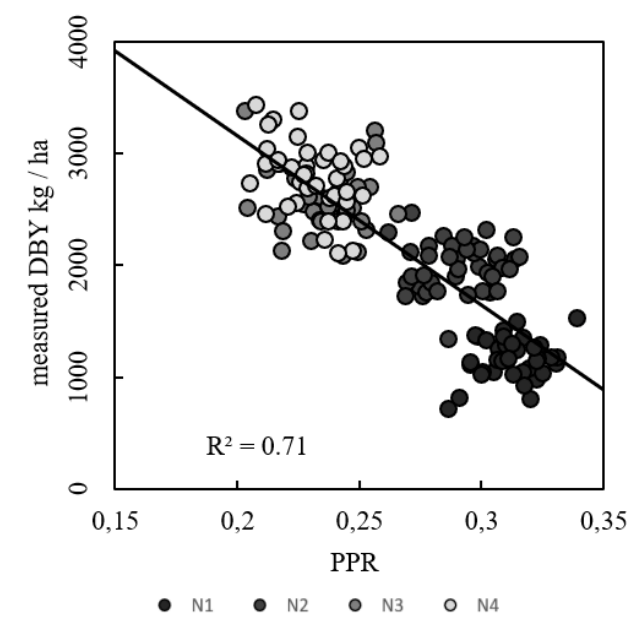

Figure 6. RGB-based Plant Pigment Ratio (PPR) vs. dry biomass yield (DBY).

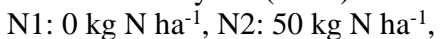
N3: $100 \mathrm{~kg} \mathrm{~N} \mathrm{ha}^{-1}$, N4: $150 \mathrm{~kg} \mathrm{~N} \mathrm{ha}^{-1}$.

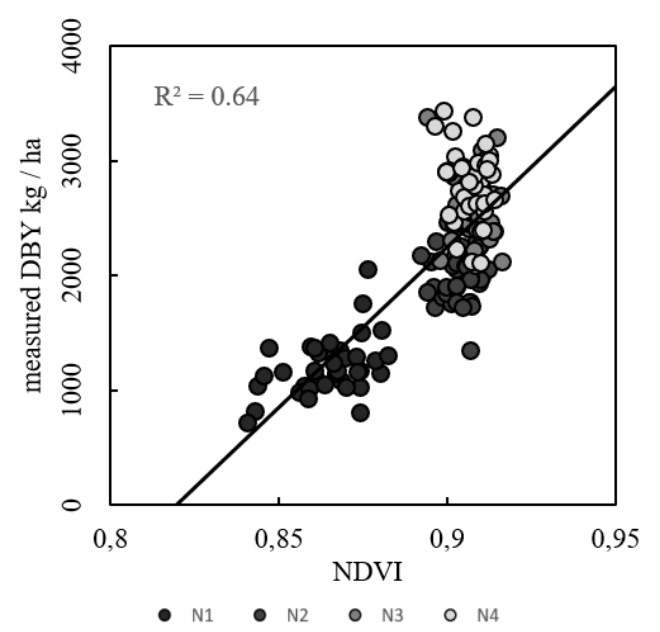

Figure 7. VNIR-based Normalized Difference Vegetation Index (NDVI) vs. dry biomass yield (DBY).

N1: $0 \mathrm{~kg} \mathrm{~N}^{-1}, \mathrm{~N} 2: 50 \mathrm{~kg} \mathrm{~N}^{-1}$,

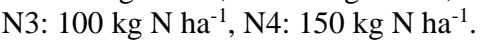

The PPR showed a strong negative relationship to DBY with an $\mathrm{R}^{2}$ of 0.71 . The four treatments are clearly visible in the scatterplot (Fig. 6). Higher DBY values are associated with lower PPR values and vice versa. The relationship of NDVI to DBY shows strong saturation effects for higher biomass values.

The cross-validation results are displayed in Figures 8, 9, and 10. 


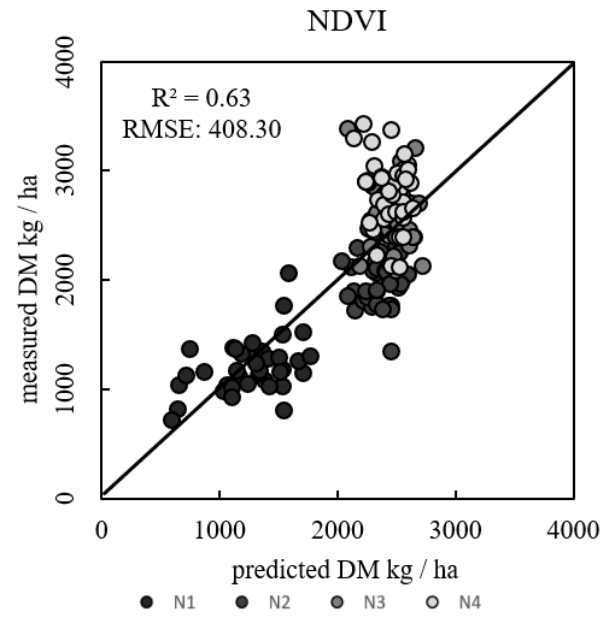

Figure 8. Measured versus predicted dry biomass yield [ $\mathrm{kg} / \mathrm{ha}]$ for NDVI.

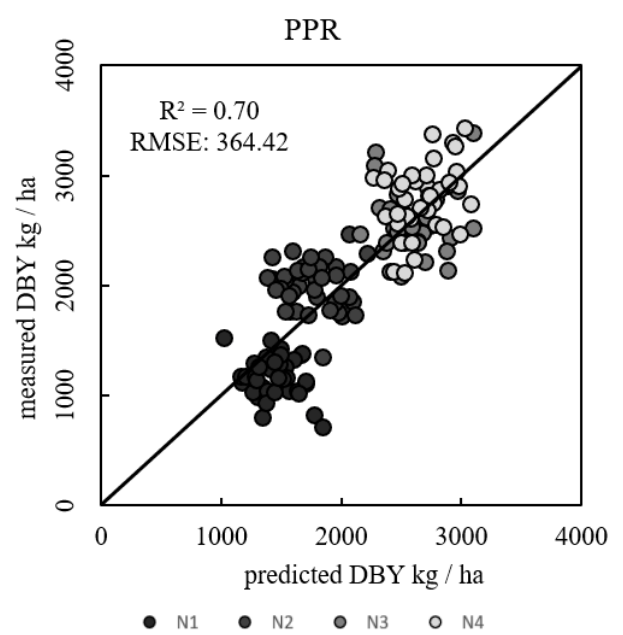

Figure 9. Measured versus predicted dry biomass yield [kg / ha] for PPR.

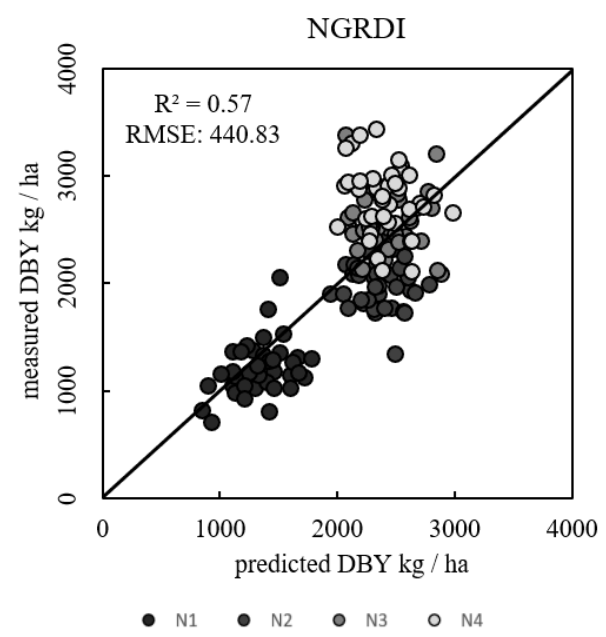

Figure 10. Measured versus predicted dry biomass yield [kg / ha] for NGRDI.

The $\mathrm{R}^{2}$ for the NDVI was 0.63 with an RMSE of $408.30 \mathrm{~kg} / \mathrm{ha}$. The PPR had a $\mathrm{R}^{2}$ of 0.70 with an RMSE of $364.42 \mathrm{~kg} / \mathrm{ha}$ and the NGRDI a $\mathrm{R}^{2}$ of 0.57 with a RMSE of $440.83 \mathrm{~kg} / \mathrm{ha}$.

The pattern between measured and predicted DBY was linear for the PPR, although there was still variation across the regression line. The pattern of the treatments was more pronounced, while $\mathrm{N} 3$ and N4 are not distinguishable.

The NGRDI cross-validation result had a similar pattern to the NDVI with saturation effects for the higher fertilized plots.

\section{DISCUSSION \& CONCLUSION}

The aim of this study was to compare simultaneously acquired high resolution RGB and VNIR vegetation indices to predict dry biomass yield in temperate grassland. The results indicate a promising approach to map grassland biomass in high spatial and temporal resolution with consumer-grade RGB cameras. The RGB-based PPR performs well as an indicator of grassland biomass. The lower DNs in the green band and the slightly higher DNs in the blue band of the higher $\mathrm{N}$ treatments are linked to carotenoids and chlorophyll. This might explain the better performance of the PPR compared to the NGRDI and NDVI. (Metternicht, 2003). The PPR can distinguish the first ( $0 \mathrm{~kg} \mathrm{~N} / \mathrm{ha})$ and second (50 kg N/ha) treatment, although it fails to distinguish between the third $(100 \mathrm{~kg} \mathrm{~N} / \mathrm{ha})$ and fourth $(150 \mathrm{~kg} \mathrm{~N} / \mathrm{ha})$ treatment, similarly to the NGRDI and NDVI. The performance of the NGRDI was similar to the results reported by Lussem et al. (2018) on the same test site, but for a different year. Hunt et al. (2005) reported a good correlation of the NGRDI to alfalfa, corn and soybean biomass, but observed saturation effects for higher biomass yield, which is similar in this study and can be related to Motohka et al. (2010), who observed that RGB-based VIs are limited to certain growing stages. Although the NDVI yields a slightly better cross-validation result than NGRDI, strong saturation effects are clearly visible.

Further investigations should be directed towards the evaluation of high-resolution RGB-based VIs to assess grassland biomass for multiple years, sites and cutting regimes. A combination of spectral features and structural features such as plant height from canopy surface models to predict grassland biomass already shows promising directions to support precision pasture management (Viljanen et al., 2018).

\section{ACKNOWLEDGEMENTS}

We acknowledge the funding by the BMBF-funded project GreenGrass (FKZ: 031B0734F) for participation at the GSW/UAVg 2019.

\section{REFERENCES}

Aasen, H., Honkavaara, E., Lucieer, A., Zarco-Tejada, P., 2018. Quantitative remote sensing at ultra-high resolution with UAV spectroscopy: A review of sensor technology, measurement procedures, and data correctionworkflows. Remote Sens. 10, 1-42. doi:10.3390/rs10071091.

FAO (Food and Agricultural Organization of the United Nations), 2010. Challenges and opportunities for carbon sequestration in grassland systems. A technical report on grassland management and climate change mitigation. Integrated Crop Management 9. doi:10.3329/jard.v7i1.4430.

Hunt, E., Cavigelli, M., Daughtry, C., McMurtrey, J., Walthall, C., 2005. Evaluation of digital photography from model aircraft for remote sensing of crop biomass and nitrogen status. Precis. Agric. 6, 359-378. doi:10.1007/s11119005-2324-5.

Kuhn, M., Wing, J., Weston, S., Williams, A., Keefer, C., Engelhardt, A., Cooper, T., Mayer, Z., Team RC, 2018. caret: Classification and Regression Training. R package version v6. 0.82 . 2017. https://cran.r- 
project.org/web/packages/caret/index.html.

Lussem, U., Bolten, A., Gnyp, M., Jasper, J., Bareth, G., 2018. Evaluation of RGB-based vegetation indices from UAV imagery to estimate forage yield in grassland. ISPRS Int. Arch. Photogramm. Remote Sens. Spat. Inf. Sci. XLII-3, 1215-1219. doi:10.5194/isprs-archives-XLII-3-12152018.

Metternicht, G., 2003. Vegetation indices derived from highresolution airborne videography for precision crop management. Int. J. Remote Sens. 24, 2855-2877. doi:10.1080/01431160210163074.

Motohka, T., Nasahara, K., Oguma, H., Tsuchida, S., 2010. Applicability of Green-Red Vegetation Index for remote sensing of vegetation phenology. Remote Sens. 2, 23692387. doi:10.3390/rs2102369.

O'Mara, F., 2012. The role of grasslands in food security and climate change. Ann. Bot. 110, 1263-1270. doi:10.1093/aob/mcs209.

Schellberg, J., Verbruggen, E., 2014. Frontiers and perspectives on research strategies in grassland technology. Crop Pasture Sci. 65, 508-523. doi:10.1071/CP13429.

Viljanen, N., Honkavaara, E., Näsi, R., Hakala, T., Niemeläinen, O., Kaivosoja, J., 2018. A novel machine learning method for estimating biomass of grass swards using a photogrammetric canopy height model, images and vegetation indices captured by a drone. Agriculture 8 . doi:10.3390/agriculture8050070.

Wachendorf, M., Fricke, T., Moeckel, T., 2017. Remote sensing as a tool to assess botanical composition, structure , quantity and quality of temperate grasslands. Grass and Forage Science, 1-14. doi:10.1111/gfs.12312.

Zhang, H., Sun, Y., Chang, L., Qin, Y., Chen, J., Qin, Y., Du, J., Yi, S., Wang, Y., 2018. Estimation of grassland canopy height and aboveground biomass at the quadrat scale using unmanned aerial vehicle. Remote Sens. 10. doi:10.3390/rs 10060851 . 